\title{
EXPLICIT EXPRESSION OF THE KRAWTCHOUK POLYNOMIAL VIA A DISCRETE GREEN'S FUNCTION
}

\author{
Gil Chun Kim and Yoonjin Lee
}

\begin{abstract}
A Krawtchouk polynomial is introduced as the classical MacWilliams identity, which can be expressed in weight-enumerator-free form of a linear code and its dual code over a Hamming scheme. In this paper we find a new explicit expression for the $p$-number and the $q$ number, which are more generalized notions of the Krawtchouk polynomial in the $P$-polynomial schemes by using an extended version of a discrete Green's function. As corollaries, we obtain a new expression of the Krawtchouk polynomial over the Hamming scheme and the Eberlein polynomial over the Johnson scheme. Furthermore, we find another version of the MacWilliams identity over a Hamming scheme.
\end{abstract}

\section{Introduction}

Let $\mathcal{C}$ be a linear code over a finite field $\mathbb{F}_{q}$ with $q$ elements of length $d$. The MacWilliams identity for linear codes over $\mathbb{F}_{q}$ is one of the most important identities in coding theory, which expresses the Hamming weight enumerator of the dual code $\mathcal{C}^{\perp}$ of a linear code $\mathcal{C}$ over $\mathbb{F}_{q}$ in terms of the Hamming weight enumerator of $\mathcal{C}$. Let $\mathbf{a}=\left(a_{0}, a_{1}, \ldots, a_{d}\right)$ (respectively, $\left.\mathbf{b}=\left(b_{0}, b_{1}, \ldots, b_{d}\right)\right)$ be the weight distributions of $\mathcal{C}$ (respectively, $\mathcal{C}^{\perp}$ ). Then MacWilliams identity can be expressed in weight-enumerator-free form as $[5,8,9]$ :

$$
\mathbf{a}=\frac{1}{\left|\mathcal{C}^{\perp}\right|} \mathbf{b}\left(p_{j}(i)\right)
$$

where $p_{j}(i)=p_{j}(i ; d, q)$ is the Krawtchouk polynomial defined by

$$
p_{j}(i)=\sum_{l=0}^{j}(-1)^{l}(q-1)^{j-l}\left(\begin{array}{l}
i \\
l
\end{array}\right)\left(\begin{array}{l}
d-i \\
j-l
\end{array}\right)(i, j=0,1, \ldots, d) .
$$

Received April 25, 2012; Revised December 18, 2012.

2010 Mathematics Subject Classification. Primary 58B34, 58J42, 81T75.

Key words and phrases. discrete Green's function, $P$-polynomial scheme, $p$-number, $q$ number, Krawtchouk polynomial, Eberlein polynomial.

The second named author was supported by Priority Research Centers Program through the NRF funded by the Ministry of Education, Science and Technology(2009-0093827) and by the NRF grant funded by the Korea government(MEST)(2012-0005432). 
Delsarte introduced the association scheme in [5]. Association schemes are important notions in algebraic graph theory and in coding theory. The classes of strongly regular graphs, distance regular graphs and symmetric circulants are instances of association schemes. In particular, the Hamming scheme and the Johnson scheme are the most important association schemes $[1,5,6]$. The Hamming scheme is mainly concerned with the distance of a code. In other words, the linear programming method produces upper bounds for the size of a code with given minimum distance, and lower bounds for the size of a design with given strength.

More generalized notions of the Krawtchouk polynomial in association schemes are the $p$-number and the $q$-number of the association schemes. One of the most important association schemes is a $P$-polynomial scheme $[5,6,8]$. In fact, the Hamming scheme and the Johnson scheme are the $P$-polynomial schemes, and the $p$-number and the $q$-number in the $P$-polynomial schemes are applied to finding some universal bounds for codes and designs $[5,6,8]$.

A Green's function is introduced in a famous essay by George Green in 1728. In [4], a discrete Green's function is defined on graphs. The Green function is closely associated with the normalized Laplacian $\mathcal{L}_{\beta}$ and is useful for solving discrete Laplace equations with boundary conditions. In $[2,3]$, F. Chung introduced the relationship between the PageRank and a discrete Green's function $\mathcal{G}_{\beta}$ with a positive real number $\beta$. A Green's function $\mathcal{G}_{\beta}$ can be explained with an inverse relation of the $\beta$-normalized Laplacian $\mathcal{L}_{\beta}$ represented by an adjacency matrix.

In this paper we find a new explicit expression (Theorem 4) for the $p$-number and the $q$-number by using an extended version of a discrete Green's function, called a normalized Green's function $\mathcal{G}_{\beta, \mathcal{N}}$; this is expressed by a basis of a nullspace of some $d \times(d+1)$ matrix $L_{s u b}$ (associated with the $P$-polynomial scheme and $\left.\mathcal{G}_{\beta, \mathcal{N}}\right)$ and the adjacency matrices of the $P$-polynomial scheme for $\beta \in \mathbb{R}$. As corollaries, we obtain a new expression of the Krawtchouk polynomial over the Hamming scheme and the Eberlein polynomial over the Johnson scheme. Furthermore, we find another version of the MacWilliams identity over a Hamming scheme (Corollary 5.6), and we also obtain another expression of the Eberlein polynomial $E_{i}(j)$ as $p$-number over the Johnson scheme $J(v, d)$ (Corollary 6.2).

In more detail, for some $\beta_{j} \in \mathbb{R}$, we show that the $j$-th column vector of the second eigenmatrix $\mathbf{Q}=\left(q_{j}(i)\right)$ is the vector $\left(u_{0}^{(j)}, u_{1}^{(j)}, \ldots, u_{d}^{(j)}\right)$ which is contained in a nullspace of some $d \times(d+1)$ matrix $L_{s u b}^{\left(\beta_{j}\right)}$ (Proposition 4.3). Furthermore, we obtain the $p$-number by using $u_{i}^{(j)}$ and the relations with the $q$ number over the association schemes (Corollary 4.4). As a main result, we show that the $p$-number $p_{j}(i)$ and the $q$-number $q_{j}(i)$ can be explicitly expressed by a determinant of a submatrix $L_{i}^{\left(\beta_{j}\right)}$ of $d \times(d+1)$ matrix $L_{s u b}^{\left(\beta_{j}\right)}$ with $\beta_{j}=\frac{p_{1}(j)}{k_{1}}-1$ $\left(j=0,1, \ldots, d, k_{1}\right.$ is a valency of $\left.R_{1}\right)$ (Theorem 4.1 ). In Corollary 5.1, we show that the Krawtchouk polynomial $p_{j}(i)$ over the Hamming scheme $H(d, q)$ can 
be explicitly expressed by a determinant of a submatrix $L_{i}^{\left(\beta_{j}\right)}$ of $d \times(d+1)$ matrix $L_{s u b}^{\left(\beta_{j}\right)}$ with $\beta_{j}=-\frac{q j}{k_{1}}(j=0,1, \ldots, d)$ and $k_{1}=d(q-1)$.

This paper is organized as follows. In Section 2, we introduce basic facts of the $P$-polynomial scheme and a Green's function $\mathcal{G}_{\beta}$. In Section 3, we introduce a $d \times(d+1)$ matrix $L_{s u b}$, and we define a normalized Green's function $\mathcal{G}_{\beta, \mathcal{N}}$. In Section 4 , for $\beta_{j} \in \mathbb{R}$, we find the relationship between a normalized Green's function $\mathcal{G}_{\beta_{j}, \mathcal{N}}$ and the $p$-number $p_{j}(i)$ (or the $q$-number $q_{j}(i)$ ). Moreover, we obtain the relationship between determinants of submatrices $L_{i}^{\left(\beta_{j}\right)}$ of $L_{s u b}^{\left(\beta_{j}\right)}$ and the $p$-number (or the $q$-number). In Section 5 , we obtain the relationship between determinants of submatrices $L_{i}^{\left(\beta_{j}\right)}$ of $L_{s u b}^{\left(\beta_{j}\right)}$ and the Krawtchouk polynomial $p_{j}(i)$. We thus obtain another version of the MacWilliams identity over $H(d, q)$. Finally, in Section 6 , we obtain the Eberlein polynomial $E_{i}(j)$ as the $p$-number over the Johnson scheme $J(v, d)$ using the determinants of submatrices $L_{i}^{\left(\beta_{j}\right)}$ of $L_{s u b}^{\left(\beta_{j}\right)}$.

\section{Preliminaries}

In this section we introduce basic facts on association schemes and the discrete Green's function.

Let $\mathfrak{X}=\left(X,\left\{R_{i}\right\}\right)(i=0,1, \ldots, d)$ be an association scheme and $d_{M}(x, y)$ be a metric over $X$. We describe the relations by their adjacency matrices $A_{i}$ $(i=0,1, \ldots, d)$ which are the $|X| \times|X|$ matrices defined by

$$
\left(A_{i}\right)_{x, y}= \begin{cases}1, & \text { if } d_{M}(x, y)=i, \\ 0, & \text { otherwise. }\end{cases}
$$

$A$ Bose Mesner algebra $\mathcal{A}$ is generated by the adjacency matrices $A_{i}$, that is, $\mathcal{A}=\left\{\sum t_{i} A_{i} \mid t_{0}, t_{1}, \ldots, t_{d} \in \mathbb{C}\right\}$. A Bose-Mesner algebra $\mathcal{A}$ has a unique basis of primitive idempotent matrices $E_{0}, E_{1}, \ldots, E_{d}$, that is,

$$
\text { (1) } E_{k} E_{l}=\delta_{k l} E_{k}(k, l=0,1, \ldots, d),(2) \sum_{i=0}^{d} E_{i}=I,
$$

where $\delta_{k l}$ is the Kronecker delta function. A Bose-Mesner algebra $\mathcal{A}$ have two basis $\left\{A_{i}\right\}$ and $\left\{E_{i}\right\}$. For $A_{i}$ and $E_{i}$, we express one in terms of the other and we obtain

$$
A_{j}=\sum_{i=0}^{d} p_{j}(i) E_{i}, E_{j}=\frac{1}{|X|} \sum_{i=0}^{d} q_{j}(i) A_{i}
$$

for $j=0,1, \ldots, d$. The $(d+1) \times(d+1)$ matrix $\mathbf{P}=\left(p_{j}(i)\right)$ (respectively, $\mathbf{Q}=$ $\left.\left(q_{j}(i)\right)\right)$ is called the first eigenmatrix (respectively, the second eigenmatrix) of the association scheme. Then $\mathbf{P}=\left(p_{j}(i)\right)$ and $\mathbf{Q}=\left(q_{j}(i)\right)$ satisfy that $q_{j}(i) / m_{j}=\overline{p_{i}(j)} / k_{i}$, where $m_{j}=\operatorname{rank}\left(E_{j}\right), k_{i}$ is the valency of $A_{i}$, and $\overline{p_{i}(j)}$ 
is the complex conjugate of $p_{i}(j)$. The adjacency matrices $A_{i}$ satisfy

$$
A_{i} A_{j}=\sum_{k=0}^{d} p_{i j}^{k} A_{k}
$$

for all $i, j$, where for $(x, y) \in R_{k}, p_{i j}^{k}$ is the number of $z \in X$ such that $(x, z) \in$ $R_{i}$ and $(z, y) \in R_{j}$. The non-negative integers $p_{i j}^{k}$ are called the intersection numbers of $\mathfrak{X}$. Let $B_{i}$ be a matrix with $(j, k)$-entries $p_{i j}^{k}$, and let $\mathcal{B}$ be an algebra spanned by $B_{0}, B_{1}, \ldots, B_{d}$. Then $B_{i}$ is called the $i$-th intersection matrix of $\mathfrak{X}$ and $\mathcal{B}$ is called the intersection algebra of $\mathfrak{X}$. In fact, the Bose-Mesner algebra $\mathcal{A}$ of $\mathfrak{X}$ is isomorphic to $\mathcal{B}$ by the map $A_{i} \rightarrow B_{i}$. In particular, $A_{i}$ and $B_{i}$ have the same minimal polynomials.

Now, we introduce definitions of the $P$-polynomial schemes and basic facts on the $P$-polynomial schemes $[1,5,6]$.

Definition. A symmetric association scheme $\mathfrak{X}=\left(X,\left\{R_{i}\right\}\right)(i=0, \ldots, d)$ is called a $P$-polynomial scheme with respect to the ordering $R_{0}, R_{1}, \ldots, R_{d}$, if there exists some complex coefficient polynomial $v_{i}(x)$ of degree $i(i=$ $0,1, \ldots, d)$ such that $A_{i}=v_{i}\left(A_{1}\right)$, where $A_{i}$ is the adjacency matrix with respect to $R_{i}$.

Let $\mathfrak{X}=\left(X,\left\{R_{i}\right\}\right)(i=0,1, \ldots, d)$ be a symmetric association scheme, and $\Gamma_{1}$ be the graph whose vertex and edge sets are $X$ and $R_{1}$ respectively. Then the following (1), (2), (3) and (4) are equivalent to each other.

(1) $\Gamma_{1}$ is a distance regular graph.

(2) The first intersection matrix $B_{1}$ is a tridiagonal matrix with non-zero off-diagonal entries,

$$
\begin{aligned}
& B_{1}=\left(\begin{array}{cccccc}
0 & k_{1} & 0 & 0 & \ldots & 0 \\
1 & a_{1} & b_{1} & 0 & \ldots & 0 \\
0 & c_{2} & a_{2} & b_{2} & \ldots & 0 \\
\vdots & \vdots & \ddots & \ddots & \ddots & \vdots \\
& & & c_{d-1} & a_{d-1} & b_{d-1} \\
0 & \ldots & \ldots & 0 & c_{d} & a_{d}
\end{array}\right) \quad\left(b_{i} \neq 0, c_{i} \neq 0\right) \\
& \text { (i) } a_{i}+b_{i}+c_{i}=k_{1}(i=0,1, \ldots, d), c_{0}=b_{d}=0 \text {, } \\
& \text { (ii) } k_{i}=\frac{k_{1} b_{1} b_{2} \cdots b_{i-1}}{c_{2} c_{3} \cdots c_{i}}(i=2,3, \ldots, d) \text {, } \\
& \text { (iii) } k_{1} \geq b_{1} \geq \cdots \geq b_{d-1} \text {, } \\
& \text { (iv) } 1 \leq c_{2} \leq \cdots \leq c_{d} \text {. }
\end{aligned}
$$

(3) $\mathfrak{X}$ is a $P$-polynomial scheme with respect to $R_{0}, R_{1}, \ldots, R_{d}$, that is, $A_{i}=v_{i}\left(A_{1}\right)(i=0,1, \ldots, d)$ for some polynomial $v_{i}(x)$ of degree $i$.

(4) First eigenmatrix $\mathbf{P}=\left(p_{j}(i)\right)$ satisfies $p_{j}(i)=v_{i}\left(\theta_{j}\right)$ for some polynomial $v_{i}(x)$ of degree $i$, where $\theta_{j}=p_{1}(j)(i, j=0,1, \ldots, d)$. 
Let $\mathfrak{X}=\left(X,\left\{R_{i}\right\}\right)(i=0,1, \ldots, d)$ be a $P$-polynomial scheme. Now, define a transition probability matrix $P$ over $\mathfrak{X}$ by

$$
P=\frac{1}{k_{1}} A_{1}
$$

where $k_{1}$ is the valency of $A_{1}$. For a function $f: X \rightarrow \mathbb{R}$, we define a Laplace operator $\Delta$ by

$$
\Delta f(x)=\frac{1}{k_{1}} \sum_{(x, y) \in R_{1}}(f(x)-f(y)) .
$$

Then, we have $\Delta=I-P=I-\frac{1}{k_{1}} A_{1}$. For all $i=0,1, \ldots, d$, a Laplace operator $\Delta$ is symmetric since $A_{1}$ is symmetric. Then, $\Delta$ is a matrix representation of $\mathcal{L}$. For $j=0,1, \ldots, d$ and orthogonal eigenfunctions $\phi_{j}^{*}$, we have

$$
\mathcal{L}=\sum_{j=0}^{d} \lambda_{j} \phi_{j}^{*} \phi_{j},
$$

where $\lambda_{j}$ is an eigenvalue of $\mathcal{L}$. Let $\mathcal{L}_{\beta}$ be the $\beta$-normalized Laplacian by $\beta I+\mathcal{L}$. For $\beta>0$, let a discrete Green's function $\mathcal{G}_{\beta}$ denote the symmetric matrix satisfying $\mathcal{L}_{\beta} \mathcal{G}_{\beta}=I$. Then we have

$$
\mathcal{G}_{\beta}=\sum_{j=0}^{d} \frac{1}{\beta+\lambda_{j}} \phi_{j}^{*} \phi_{j}
$$

For $\beta>0$, we have

$$
\mathcal{G}_{\beta}(\beta I+I-P)=I,
$$

that is,

$$
\mathcal{G}_{\beta}=((\beta+1) I-P)^{-1} .
$$

Thus, this implies that

$$
\begin{aligned}
\mathcal{L}_{\beta} & =(\beta+1) I-P=(\beta+1) I-\frac{1}{k_{1}} A_{1} \\
& =(\beta+1) I-\frac{1}{k_{1}} \sum_{j=0}^{d} p_{1}(j) E_{j} .
\end{aligned}
$$

Since $I=E_{0}+E_{1}+\cdots+E_{d}$, we have

$$
\begin{aligned}
\mathcal{L}_{\beta} & =(\beta+1)\left(E_{0}+E_{1}+\cdots+E_{d}\right)-\frac{1}{k_{1}}\left(p_{1}(0) E_{0}+p_{1}(1) E_{1}+\cdots+p_{1}(d) E_{d}\right) \\
& =\sum_{j=0}^{d}\left(\beta+1-\frac{1}{k_{1}} p_{1}(j)\right) E_{j},
\end{aligned}
$$


where $\beta+1-\frac{1}{k_{1}} p_{1}(j)$ is an eigenvalue of $\mathcal{L}_{\beta}$. Hence, a Green's function $\mathcal{G}_{\beta}$ can be expressed by

$$
\mathcal{G}_{\beta}=\sum_{j=0}^{d}\left(\frac{k_{1}}{(\beta+1) k_{1}-p_{1}(j)}\right) E_{j} .
$$

Since $E_{j}=(1 /|X|) \sum q_{j}(i) A_{i}$, the Green function $\mathcal{G}_{\beta}$ is a linear combination of adjacency matrices $A_{i}$ as follows:

$$
\mathcal{G}_{\beta}=r_{0} A_{0}+r_{1} A_{1}+\cdots+r_{d} A_{d}
$$

for some $r_{i}(i=0,1, \ldots, d)$.

The following notations are used throughout this paper.

- $\mathbf{P}$ : the first eigenmatrix of the $P$-polynomial scheme.

- $\mathbf{Q}$ : the second eigenmatrix of the $P$-polynomial scheme.

- $p_{j}(i):(i, j)$-component of $\mathbf{P}$.

- $q_{j}(i):(i, j)$-component of $\mathbf{Q}$.

- $k_{i}: i$-th the valency of the $P$-polynomial scheme.

- $m_{j}: j$-th the multiplicity of the $P$-polynomial scheme.

- $\mathcal{N}(A)$ : a nullspace of a matrix $A$.

- $\Gamma_{1}$ : a graph with respect to $R_{1}$ of a $P$-polynomial scheme.

\section{A reduction matrix $L_{s u b}$ on $\mathcal{L}_{\beta}$ of the $P$-polynomial scheme}

In this section we first introduce a $d \times(d+1)$ matrix $L_{s u b}$ obtained from $\mathcal{L}_{\beta}$. Then we show that $L_{\text {sub }}$ is closely related to the discrete Green's function $\mathcal{G}_{\beta}$ in the following lemma.

Lemma 3.1. For $\beta>0$, a Green's function $\mathcal{G}_{\beta}$ is expressed as

$$
\mathcal{G}_{\beta}=c u_{0} A_{0}+c u_{1} A_{1}+\cdots+c u_{d} A_{d}
$$

for some nonzero $c \in \mathbb{R}$, where $\left(u_{0}, u_{1}, \ldots, u_{d}\right)$ is the unique basis of the nullspace $\mathcal{N}\left(L_{\text {sub }}\right)$ of $L_{\text {sub }}$ with $u_{d}=1$.

Proof. Let $\mathfrak{X}=\left(X,\left\{R_{i}\right\}\right)(i=0,1, \ldots, d)$ be a $P$-polynomial scheme with respect to a matric $d_{M}(x, y)$ for $x, y \in X$. Let $L$ be a $(|X|-1) \times|X|$ matrix obtained by the removal of the first row of $\mathcal{L}_{\beta}=(\beta+1) I-\frac{1}{k_{1}} A_{1}$. Then we have the rank of $L$ is $|X|-1$, and the nullity is 1 since $\mathcal{G}_{\beta}$ has the inverse matrix. A basis of the nullspace of $L$ can be induced from $r_{k}$ 's which are coefficients of $A_{k}$ in $\mathcal{G}_{\beta}$. Let $\mathcal{G}_{\beta}^{(1)}$ be the first column vector of $\mathcal{G}_{\beta}$ which is arranged in the order $r_{0}, r_{1}, \ldots, r_{d}$. Then $\mathcal{G}_{\beta}^{(1)}$ is a $|X| \times 1$ matrix, and we have

$$
L \mathcal{G}_{\beta}^{(1)}=O
$$

since $\mathcal{G}_{\beta}$ is orthogonal. Since the Bose-Mesner algebra $\mathcal{A}$ is isomorphic to the intersection algebra $\mathcal{B}$ of $\mathfrak{X}, \mathcal{G}_{\beta}^{-1}=(\beta+1) I-\frac{1}{k_{1}} A_{1}$ is corresponding with $(\beta+1) I-\frac{1}{k_{1}} B_{1}$. Let $L^{\prime}$ be a $(d+1) \times(d+1)$ matrix as $-k_{1}\left((\beta+1) I-\frac{1}{k_{1}} B_{1}\right)$, 
that is, $L^{\prime}=B_{1}-k_{1}(\beta+1) I$. Let $L_{\text {sub }}$ be a $d \times(d+1)$ matrix obtained by the removal of the first row of $L^{\prime}$. Then, we obtain $L_{s u b}$ as follows:

$$
L_{s u b}=\left(\begin{array}{ccccccc}
c_{1} & s_{1} & b_{1} & 0 & 0 & \cdots & 0 \\
0 & c_{2} & s_{2} & b_{2} & 0 & \cdots & 0 \\
0 & 0 & c_{3} & s_{3} & b_{3} & \cdots & 0 \\
\vdots & \vdots & \vdots & \ddots & \ddots & \ddots & \vdots \\
0 & 0 & 0 & \cdots & c_{d-1} & s_{d-1} & b_{d-1} \\
0 & 0 & 0 & \cdots & 0 & c_{d} & s_{d}
\end{array}\right) \text {, }
$$

where $s_{i}=a_{i}-k_{1}(\beta+1)$ for $i=1,2, \ldots, d$ and $a_{i}, b_{i}, c_{i}$ are as the entries of $B_{1}$ in Section 2. Therefore, by (3), we have

$$
L_{s u b}\left(\begin{array}{c}
r_{0} \\
r_{1} \\
\vdots \\
r_{d}
\end{array}\right)=O .
$$

Since $1=c_{1} \leq c_{2} \leq \cdots \leq c_{d}, \operatorname{rank}\left(L_{s u b}\right)=d$ and $\operatorname{dim} \mathcal{N}\left(L_{s u b}\right)=1$. Then from Eq. (1), we obtain the result as desired.

A Green's function $\mathcal{G}_{\beta}$ is defined only for $\beta>0$, and $\mathcal{G}_{\beta}$ is expressed as a linear combination of adjacency matrices $A_{i}$ such as in Eq.(2). But, for $\beta \leq 0$, $\mathcal{G}_{\beta}$ may be a singular matrix, so there is no Green's function notion for this case. We, however, still have $\operatorname{rank}\left(L_{\text {sub }}\right)=d$ for $\beta \in \mathbb{R}$, so we can obtain a unique basis $\left(u_{0}, u_{1}, \ldots, u_{d}\right)$ of $\mathcal{N}\left(L_{s u b}\right)$ with $u_{d}=1$. In this context, we extend a notion of a Green's function $\mathcal{G}_{\beta}$ associated with any real number $\beta$ as follows. The following definition plays an important role for computation of the $p$-number and the $q$-number as we will see in Section 4.

Definition. For $\beta \in \mathbb{R}$, let $\left(u_{0}, u_{1}, \ldots, u_{d}\right) \in \mathcal{N}\left(L_{s u b}\right)$ with $u_{d}=1$ and let $\mathcal{G}_{\beta, \mathcal{N}}=c u_{0} A_{0}+c u_{1} A_{1}+\cdots+c u_{d} A_{d}$, where $c$ is some nonzero $\in \mathbb{R}$ if $\beta>0$ and $c=1$ if $\beta \leq 0$. Then $\mathcal{G}_{\beta, \mathcal{N}}$ is called the normalized Green's function.

The $P$-polynomial schemes are defined by Delsarte. We know the two most important examples, namely the Hamming scheme $H(d, q)$ and the Johnson scheme $J(v, d)$. In the following example, we show a normalized Green's function over a Hamming scheme $H(5,3)$.

Example 3.2. Let $H(5,3)$ be a Hamming scheme over $\mathbb{F}_{3}^{5}$. Choosing $\beta=-\frac{1}{10}$, we obtain a $5 \times 6$ matrix $L_{s u b}$ as follows:

$$
\left(\begin{array}{cccccc}
1 & -8 & 8 & 0 & 0 & 0 \\
0 & 2 & -7 & 6 & 0 & 0 \\
0 & 0 & 3 & -6 & 4 & 0 \\
0 & 0 & 0 & 4 & -5 & 2 \\
0 & 0 & 0 & 0 & 5 & -4
\end{array}\right)
$$


Also, a basis of $\mathcal{N}\left(L_{s u b}\right)$ is $\left(-\frac{40}{3},-\frac{26}{15},-\frac{1}{15}, \frac{1}{2}, \frac{4}{5}, 1\right)$. Thus we obtain a normalized Green's function $\mathcal{G}_{\beta, \mathcal{N}}$ as follows:

$$
\mathcal{G}_{\beta, \mathcal{N}}=-\frac{40}{3} A_{0}-\frac{26}{15} A_{1}=\frac{1}{15} A_{2}+\frac{1}{2} A_{3}+\frac{4}{5} A_{4}+A_{5}
$$

where $A_{i}(i=0,1, \ldots, 5)$ are the adjacency matrices of $H(5,3)$.

\section{Relation between the $p$-number (or the $q$-number) and the normalized Green's function}

In this section, for $\beta \in R$, we find the relationship between a normalized Green's function $\mathcal{G}_{\beta, \mathcal{N}}$ and the $p$-number $p_{j}(i)$ (or the $q$-number $q_{j}(i)$ ) over the $P$-polynomial scheme. In fact, the goal of this section is to prove the following main result, which shows a new expression of the $p$-number (or the $q$-number) over the $P$-polynomial scheme.

Theorem 4.1. Let $\mathfrak{X}=\left(X,\left\{R_{i}\right\}\right)(i=0,1, \ldots, d)$ be a P-polynomial scheme, and let $\mathbf{P}=\left(p_{j}(i)\right)$ (respectively, $\left.\mathbf{Q}=\left(q_{j}(i)\right)\right)$ be the first eigenmatrix (respectively, the second eigenmatrix $)$ of $\mathfrak{X}$. Then, for $\beta_{j}=\frac{p_{1}(j)}{k_{1}}-1(j=0,1, \ldots, d)$, we have

$$
\begin{aligned}
& p_{0}(j)=k_{0}, q_{j}(0)=m_{j}, \\
& p_{i}(j)=(-1)^{i} k_{i} c_{1} c_{2} \cdots c_{i} \frac{\operatorname{det}\left(L_{i}^{\left(\beta_{j}\right)}\right)}{\operatorname{det}\left(L_{0}^{\left(\beta_{j}\right)}\right)}(i=1,2, \ldots, d), \\
& q_{j}(i)=(-1)^{i} m_{j} c_{1} c_{2} \cdots c_{i} \frac{\operatorname{det}\left(L_{i}^{\left(\beta_{j}\right)}\right)}{\operatorname{det}\left(L_{0}^{\left(\beta_{j}\right)}\right)}(i=1,2, \ldots, d),
\end{aligned}
$$

where $m_{j}=\operatorname{rank}\left(E_{j}\right)$ and $k_{i}$ is a valency of $R_{i}$.

For the proof of Theorem 4.1, we need Proposition 4.3, Corollary 4.4 and Lemma 4.5.

We need the following lemma for Proposition 4.3.

Lemma 4.2. Let $\mathfrak{X}=\left(X,\left\{R_{i}\right\}\right)(i=0,1, \ldots, d)$ be a P-polynomial scheme and let $L_{\text {sub }}$ be a matrix of $\mathfrak{X}$ as in Section 3. Let $u=\left(u_{0}, u_{1}, \ldots, u_{d}\right)$ be a $(d+1)$ - vector. Then $u \in \mathcal{N}\left(L_{\text {sub }}\right)$ if and only if

$$
u^{\prime}:=(\underbrace{u_{0}}_{k_{0}}, \underbrace{u_{1}, \ldots, u_{1}}_{k_{1}}, \ldots, \underbrace{u_{d}, \ldots, u_{d}}_{k_{d}}) \in \mathcal{N}(L),
$$

where $L$ is a $(|X|-1) \times|X|$ matrix on (3) in Section 3.

Proof. $(\Rightarrow)$ Let $u$ be a basis of $\mathcal{N}\left(L_{s u b}\right)$, and let $l_{i}$ be the $i$-th row vector of $L_{s u b}$ for $i=1,2, \ldots, d$. Then $l_{i}$ is $\left(0, \ldots, 0, i, s_{i}, t_{i}, 0, \ldots 0\right)$. Since $u \cdot l_{i}=0$,

$$
\begin{gathered}
\left(u_{i-1}\right)\left(c_{i}\right)+\left(u_{i}\right)\left(s_{i}\right)+\left(u_{i+1}\right)\left(t_{i}\right)=0 \\
\Leftrightarrow-\frac{1}{k_{1}}\left(\left(u_{i-1}\right)\left(c_{i}\right)+\left(u_{i}\right)\left(s_{i}\right)+\left(u_{i+1}\right)\left(t_{i}\right)\right)=0 .
\end{gathered}
$$


Thus, the row vector of $L$ is orthogonal to $u^{\prime}$. That is, $u^{\prime} \in \mathcal{N}(L)$.

$(\Leftarrow)$ It is clear by the previous process of obtaining $L_{s u b}$ from $L$ in Section 3 .

For some $\beta_{j} \in \mathbb{R}$, let $L_{\text {sub }}^{\left(\beta_{j}\right)}$ be a $d \times(d+1)$ matrix as $L_{s u b}$ in Section 3 , and let $\left(u_{0}^{(j)}, u_{1}^{(j)}, \ldots, u_{d}^{(j)}\right)$ be a basis of $\mathcal{N}\left(L_{s u b}^{\left(\beta_{j}\right)}\right)$.

Let $\mathfrak{X}=\left(X,\left\{R_{i}\right\}\right)(i=0,1, \ldots, d)$ be a $P$-polynomial scheme, and let $P=\left(p_{j}(i)\right)$ (respectively, $\left.Q=\left(q_{j}(i)\right)\right)$ be the first eigenmatrix (respectively, the second eigenmatrix) of $\mathfrak{X}$. In the following theorem, we show that the $j$-th column vector of the second eigenmatrix $\mathbf{Q}=\left(q_{j}(i)\right)$ belongs to $\mathcal{N}\left(L_{\text {sub }}^{\left(\beta_{j}\right)}\right)$ for $\beta_{j}=\frac{p_{1}(j)}{k_{1}}-1(j=0,1, \ldots, d)$. That is, we find the relationship between $u_{i}^{(j)}$ and component $q_{j}(i)$ of the second eigenmatrix $\mathbf{Q}=\left(q_{j}(i)\right)$ over the $P$ polynomial scheme.

Proposition 4.3. Let $\mathfrak{X}=\left(X,\left\{R_{i}\right\}\right)(i=0,1, \ldots, d)$ be a P-polynomial scheme. For $\beta_{j}=\frac{p_{1}(j)}{k_{1}}-1(j=0,1, \ldots, d)$, let $\mathcal{G}_{\beta_{j}, \mathcal{N}}=u_{0}^{(j)} A_{0}+u_{1}^{(j)} A_{1}+$ $\ldots+u_{d}^{(j)} A_{d}$ be a normalized Green's function with $u_{d}^{(j)}=1$. Then $q_{j}(i)$ satisfy $q_{j}(i)=m_{j} \frac{u_{i}^{(j)}}{u_{0}^{(j)}}(i=0,1, \ldots, d)$. That is, the $j$-th column of the second eigenmatrix $\mathbf{Q}=\left(q_{j}(i)\right)$ is equal to $\frac{m_{j}}{u_{0}^{(j)}}\left(u_{0}^{(j)}, u_{1}^{(j)}, \ldots, u_{d}^{(j)}\right)^{T}$, where $m_{j}$ is the $j$-th multiplicity of $\mathfrak{X}$.

Proof. Since $\beta_{j} I+\mathcal{L}=\sum\left(\left(\beta_{j}+1\right)-\frac{1}{k_{1}} p_{1}(j)\right) E_{j}=\sum\left[\left(\left(\beta_{j}+1\right) k_{1}-p_{1}(j)\right) / k_{1}\right] E_{j}$, $\left(\left(\beta_{j}+1\right) k_{1}-p_{1}(j)\right) / k_{1}$ are eigenvalues of $\beta_{j} I+\mathcal{L}$, where $\beta_{j}=\frac{p_{1}(i)}{k_{1}}-1$ satisfies $\left(\beta_{j}+1\right) k_{1}-p_{1}(j)=0$. So we have $\left(\beta_{j} I+\mathcal{L}\right) E_{j}=O$. Therefore, every row vector of $L$ in Section 3 is orthogonal to every column vector of $E_{j}$. Since $E_{j}=\frac{1}{|X|} \sum q_{j}(i) A_{i}$, the first column vector of $E_{j}$ is a component of $\mathcal{N}(L)$ and can be written as

$$
\frac{1}{|X|}\left(q_{j}(0), q_{j}(1), \ldots, q_{j}(1), q_{j}(2), \ldots, q_{j}(2), \ldots, q_{j}(d)\right)^{T} .
$$

Thus, by Lemma $4.2,\left(q_{j}(0), q_{j}(1), \ldots, q_{j}(d)\right) \in \mathcal{N}\left(L_{\text {sub }}^{\left(\beta_{j}\right)}\right)$. Since $\operatorname{dim}\left(\mathcal{N}\left(L_{\text {sub }}^{\left(\beta_{j}\right)}\right)\right)$ $=1$, for $\beta_{j}=\frac{p_{1}(j)}{k_{1}}-1(j=0,1, \ldots, d)$, the $j$-th column vectors of the second eigenmatrix $\mathbf{Q}=\left(q_{j}(i)\right)$ are equal to $q_{j}(d)\left(u_{0}^{(j)}, u_{1}^{(j)}, \ldots, u_{d}^{(j)}\right)^{T}$ with respect to a basis $\left(u_{0}^{(j)}, u_{1}^{(j)}, \ldots, u_{d}^{(j)}\right)$ of $\mathcal{N}\left(L_{s u b}^{\left(\beta_{j}\right)}\right)$ with $u_{d}^{(j)}=1$. Moreover, we have $q_{j}(i)=q_{j}(d) u_{i}^{(j)}$. Since $q_{j}(0)=m_{j}$, we have $q_{j}(d)=\frac{m_{j}}{u_{0}^{(j)}}$.

Corollary 4.4. Let $\mathfrak{X}=\left(X,\left\{R_{i}\right\}\right)(i=0,1, \ldots, d)$ be a P-polynomial scheme and for $\beta_{j}=\frac{p_{1}(j)}{k_{1}}-1(j=0,1, \ldots, d)$, let $\mathcal{G}_{\beta_{j}, \mathcal{N}}=u_{0}^{(j)} A_{0}+u_{1}^{(j)} A_{1}+\cdots+u_{d}^{(j)} A_{d}$ be a normalized Green's function. Then $p_{i}(j)$ and $u_{i}^{(j)}$ satisfy $p_{i}(j)=k_{i} \frac{u_{i}^{(j)}}{u_{0}^{(j)}}$. 
Proof. By Proposition 4.3, we know $q_{j}(i)=m_{j} \frac{u_{i}^{(j)}}{u_{0}^{(j)}}$. Since $\mathfrak{X}$ is a symmetric association scheme, we have $\overline{p_{i}(j)}=p_{i}(j)$. Also, we know the $p$-number and the $q$-number satisfy $q_{j}(i) / m_{j}=\overline{p_{i}(j)} / k_{i}$. Thus we have

$$
\frac{m_{j}}{k_{i}} p_{i}(j)=m_{j} \frac{u_{i}^{(j)}}{u_{0}^{(j)}} \Leftrightarrow p_{i}(j)=k_{i} \frac{u_{i}^{(j)}}{u_{0}^{(j)}},
$$

where $m_{j}=\operatorname{rank}\left(E_{j}\right)$ and $k_{i}$ is a valency of $R_{i}$.

Let $\left(u_{0}, u_{1}, \ldots, u_{d}\right)$ be a basis of $\mathcal{N}\left(L_{s u b}\right)$ with $u_{d}=1$. In the following Lemma, for $\beta \in \mathbb{R}$, we find an explicit expression of $u_{i}$ by a determinant of a submatrix $L_{i}$ of $L_{s u b}$ as in Section 3. Thus, by Proposition 4.3 and Corollary 4.4 , the $p$-number $p_{j}(i)$ and the $q$-number $q_{j}(i)$ are expressed by a determinant of a submatrix of $L_{s u b}^{\left(\beta_{j}\right)}$.

Lemma 4.5. For $\beta \in \mathbb{R}$, let $L_{0}$ be a $d \times d$ matrix obtained by the removal of the first column of $L_{\text {sub }}$. Let $L_{i}$ be $a(d-i) \times(d-i)$ matrix obtained by the removal from the first row (respectively, column) to the $i$-th row (respectively, column) of $L_{0}$, and let $\left(u_{0}, u_{1}, \ldots, u_{d}\right)$ be a basis of $\mathcal{N}\left(L_{s u b}\right)$ with $u_{d}=1$. Then

$$
u_{i}=(-1)^{d-i} \frac{\operatorname{det}\left(L_{i}\right)}{c_{i+1} c_{i+2} \cdots c_{d}}, i=0,1, \ldots, d-1,
$$

where $L_{\text {sub }}$ is defined as in Section 3 and $\operatorname{det}\left(L_{d}\right)=1$.

Proof. Since $L_{d-1}$ is a $1 \times 1$ matrix, thus by (4) in Section 3 , we have

$$
\operatorname{det}\left(L_{d-1}\right)=s_{d}
$$

Thus, we have

$$
u_{d-1}=-\frac{s_{d}}{c_{d}}=(-1)^{d-d+1} \frac{\operatorname{det}\left(L_{d-1}\right)}{c_{d}} .
$$

Similarly, we have $u_{d-2}=(-1)^{d-d+2} \frac{\operatorname{det}\left(L_{d-2}\right)}{c_{d-1} c_{d}}$.

Next, we show the following: for $i=3, \ldots, d$,

$$
u_{d-i}=(-1)^{i} \frac{\operatorname{det}\left(L_{d-i}\right)}{c_{d-i+1} c_{d-i+2} \cdots c_{d}} .
$$

We consider an $i \times i$ submatrix $T$ which is obtained from $L_{s u b}$ by applying some elementary operations as follows:

$$
\left(\begin{array}{ccccc}
A & B & 0 & \cdots & 0 \\
1 & 0 & 0 & \cdots & -u_{d-i+1} \\
0 & 1 & 0 & \cdots & -u_{d-i+2} \\
\vdots & & \ddots & & \vdots \\
0 & \cdots & 0 & 1 & -u_{d-1}
\end{array}\right)
$$


where $A=\frac{s_{d-i+1}}{c_{d-i+1}}$ and $B=\frac{t_{d-i+1}}{c_{d-i+1}}$. Then $\operatorname{det}(T)=(-1)^{i}\left(-u_{d-i+1}\right) A-$ $(-1)^{i+1}\left(-u_{d-i+2}\right) B$, and $\operatorname{det}\left(L_{d-i}\right)$ is as follows:

$$
\begin{aligned}
\operatorname{det}\left(L_{d-i}\right) & =c_{d-i+1} \cdots c_{d-1} c_{d} \operatorname{det}(T) \\
& =(-1)^{i+1} c_{d-i+1} \cdots c_{d-1} c_{d}\left(u_{d-i+1} A+u_{d-i+2} B\right) \\
& =(-1)^{i+1} c_{d-i+1} \cdots c_{d-1} c_{d}\left(-u_{d-i}\right) \\
\Leftrightarrow u_{d-i} & =(-1)^{i} \frac{\operatorname{det}\left(L_{d-i}\right)}{c_{d-i+1} \cdots c_{d-1} c_{d}} .
\end{aligned}
$$

Therefore, we have

$$
u_{i}=(-1)^{d-i} \frac{\operatorname{det}\left(L_{i}\right)}{c_{i+1} c_{i+2} \cdots c_{d}}\left(i=0,1, \ldots, d-1, \operatorname{det}\left(L_{d}\right)=1\right) .
$$

For $\beta_{j}=\frac{p_{1}(j)}{k_{1}}-1$, let $\mathcal{G}_{\beta, \mathcal{N}}=u_{0}^{(j)} A_{0}+u_{1}^{(j)} A_{1}+\cdots+u_{d}^{(j)} A_{d}$ be a normalized Green's function. Then, by Proposition 4.3, Corollary 4.4 and Lemma 4.5, the $p$-number $p_{j}(i)$ and the $q$-number $q_{j}(i)$ are expressed by a determinant of a submatrix of $L_{\text {sub }}^{\left(\beta_{j}\right)}$. Let $L_{i}^{\left(\beta_{j}\right)}$ be a $(d-i) \times(d-i)$ submatrix of $L_{\text {sub }}^{\left(\beta_{j}\right)}$ for $\beta_{j}=\frac{p_{1}(j)}{k_{1}}-1(j=0,1, \ldots, d)$ as $L_{i}$ in Lemma 4.5. Then we obtain Theorem 4.1, the main result of this paper, by Proposition 4.3, Corollary 4.4 and Lemma 4.5 .

The proof of Theorem 4.1. By Proposition 4.3 and Corollary 4.4, $p_{i}(j)=k_{i} \frac{u_{i}^{(j)}}{u_{0}^{(j)}}$ and $q_{j}(i)=m_{j} \frac{u_{i}^{(j)}}{u_{0}^{(j)}}$. Thus we have

$$
p_{0}(j)=k_{0}, q_{j}(0)=m_{j} .
$$

Also, by Lemma 4.5 , we have $(i=1,2, \ldots, d)$

$$
\begin{aligned}
& p_{i}(j)=k_{i} \frac{u_{i}^{(j)}}{u_{0}^{(j)}}=k_{i} \frac{(-1)^{d-i} \frac{\operatorname{det}\left(L_{i}^{\left(\beta_{j}\right)}\right)}{c_{i+1} c_{i+2} \cdots c_{d}}}{(-1)^{d} \frac{\operatorname{det}\left(L_{0}^{\left(\beta_{j}\right)}\right)}{c_{1} c_{2} \cdots c_{d}}}=(-1)^{i} k_{i} c_{1} c_{2} \cdots c_{i} \frac{\operatorname{det}\left(L_{i}^{\left(\beta_{j}\right)}\right)}{\operatorname{det}\left(L_{0}^{\left(\beta_{j}\right)}\right)} \\
& q_{j}(i)=m_{j} \frac{u_{i}^{(j)}}{u_{0}^{(j)}}=m_{j} \frac{(-1)^{d-i} \frac{\operatorname{det}\left(L_{i}^{\left(\beta_{j}\right)}\right)}{c_{i+1} c_{i+2} \cdots c_{d}}}{(-1)^{d} \frac{\operatorname{det}\left(L_{0}^{\left(\beta_{j}\right)}\right)}{c_{1} c_{2} \cdots c_{d}}}=(-1)^{i} m_{j} c_{1} c_{2} \cdots c_{i} \frac{\operatorname{det}\left(L_{i}^{\left(\beta_{j}\right)}\right)}{\operatorname{det}\left(L_{0}^{\left(\beta_{j}\right)}\right)}
\end{aligned}
$$

where $\beta_{j}=\frac{p_{1}(j)}{k_{1}}-1(j=0,1, \ldots, d)$.

In [7], we have a method of computation for determinants of tridiagonal matrices, which we apply here to $L_{i}^{\left(\beta_{j}\right)}$.

Remark 4.6. Since $L_{i}^{\left(\beta_{j}\right)}$ is a tridiagonal matrix, the determinant of $L_{i}^{\left(\beta_{j}\right)}$ can be evaluated by multiplication of $2 \times 2$ matrices. Let $L_{i}^{\left(\beta_{j}\right)}$ be a $(d-i) \times(d-i)$ 
matrix as follows : $(i=0,1, \ldots, d-2)$

$$
\left(\begin{array}{cccc}
s_{i+1} & b_{i+1} & & \\
c_{i+2} & \ddots & \ddots & \\
& \ddots & \ddots & b_{d-1} \\
& & c_{d} & s_{d}
\end{array}\right),
$$

where $s_{k}=a_{k}-k_{1}\left(\beta_{j}+1\right)(k=1, \ldots, d)$ and $a_{k}, b_{k}, c_{k}$ are as in (4). Thus, the determinant of $L_{i}^{\left(\beta_{j}\right)}$ is

$$
\begin{aligned}
& \operatorname{tr}\left[\left(\begin{array}{cc}
s_{d} & -b_{d-1} c_{d} \\
1 & 0
\end{array}\right) \cdots\left(\begin{array}{cc}
s_{i+2} & -b_{i+1} c_{i+2} \\
1 & 0
\end{array}\right)\left(\begin{array}{cc}
s_{i+1} & 0 \\
1 & 0
\end{array}\right)\right] \\
= & \operatorname{tr}\left[\prod_{l=0}^{d-i-2}\left(\begin{array}{cc}
s_{d-l} & -b_{d-l-1} c_{d-l} \\
1 & 0
\end{array}\right) \times\left(\begin{array}{cc}
s_{i+1} & 0 \\
1 & 0
\end{array}\right)\right] \\
= & \operatorname{det}\left(L_{i}^{\left(\beta_{j}\right)}\right) .
\end{aligned}
$$

\section{The Krawtchouk polynomial of the Hamming scheme on the normalized Green's function}

Let $H(d, q)$ be a Hamming scheme over $\mathbb{F}_{q}^{d}$. Since the Hamming scheme is a self-dual scheme, $\mathbf{P}=\left(p_{j}(i)\right)$ is equal to $\mathbf{Q}=\left(q_{j}(i)\right)$. The $p$-number $p_{j}(i)$ of a Hamming scheme $H(d, q)$ is defined by the Krawtchouk polynomial. Thus, we have

$$
\begin{aligned}
p_{1}(j) & =(-1)^{0}(q-1)\left(\begin{array}{l}
j \\
0
\end{array}\right)\left(\begin{array}{c}
d-j \\
1
\end{array}\right)+(-1)(q-1)^{1-1}\left(\begin{array}{l}
j \\
1
\end{array}\right)\left(\begin{array}{l}
d-j \\
1-1
\end{array}\right) \\
& =d(q-1)-q j .
\end{aligned}
$$

Also, $k_{1}=d(q-1)$. Then $\beta_{j}=\frac{p_{1}(j)}{k_{1}}-1=\frac{d(q-1)-q j-d(q-1)}{d(q-1)}=-\frac{q j}{d(q-1)}$. Thus, for $\beta_{j}=-\frac{q j}{d(q-1)}$, we have a matrix $L_{s u b}^{\left(\beta_{j}\right)}$ as follows:

$$
L_{s u b}^{\left(\beta_{j}\right)}=\left(\begin{array}{ccccccc}
1 & s_{1} & t_{1} & 0 & 0 & \cdots & 0 \\
0 & 2 & s_{2} & t_{2} & 0 & \cdots & 0 \\
0 & 0 & 3 & s_{3} & t_{3} & \cdots & 0 \\
\vdots & \vdots & \vdots & \ddots & \ddots & \ddots & 0 \\
0 & 0 & 0 & \cdots & d-1 & s_{d-1} & t_{d-1} \\
0 & 0 & 0 & \cdots & 0 & d & s_{d}
\end{array}\right),
$$

where $s_{i}=i(q-2)-d(q-1)\left(\beta_{j}+1\right)$ for $i=1,2, \ldots, d$, and $t_{k}=(d-k)(q-1)$ for $k=1,2, \ldots, d-1$.

In the following Corollary 5.1, we show that the Krawtchouk polynomial $p_{j}(i)$ can be explicitly computed by determinants of submatrices of $L_{\text {sub }}^{\left(\beta_{j}\right)}$ associate with a Green's function. 
Corollary 5.1. Let $H(d, q)$ be a Hamming scheme over $\mathbb{F}_{q}^{d}$. Let $L_{i}^{\left(\beta_{j}\right)}$ be a $(d-i) \times(d-i)$ submatrix of $L_{\text {sub }}^{\left(\beta_{j}\right)}$ for $\beta_{j}=-\frac{q j}{d(q-1)}(j=0,1, \ldots, d)$ as $L_{i}$ in Lemma 4.5. Then, we have

$$
p_{j}(i)=p_{j}(d) u_{i}^{(j)}=(-1)^{d+j-i}\left(\begin{array}{l}
d \\
j
\end{array}\right) \frac{i ! \operatorname{det}\left(L_{i}^{\left(\beta_{j}\right)}\right)}{d !}
$$

for $i, j=0,1, \ldots, d$.

Proof. The Hamming scheme is a self-dual scheme. That is, $\mathbf{P}=\mathbf{Q}\left(p_{j}(i)=\right.$ $\left.q_{j}(i)\right)$. Thus, by proof of Proposition 4.3 , we have $p_{j}(i)=p_{j}(d) u_{i}^{(j)}$. Since $p_{j}(d)=(-1)^{j}\left(\begin{array}{l}d \\ j\end{array}\right)$ and by Lemma 4.5 ,

$$
p_{j}(i)=(-1)^{d+j-i}\left(\begin{array}{l}
d \\
j
\end{array}\right) \frac{i ! \operatorname{det}\left(L_{i}^{\left(\beta_{j}\right)}\right)}{d !} .
$$

Moreover, by Theorem 4.1 and Corollary 5.1,

$$
\operatorname{det}\left(L_{0}^{\left(\beta_{j}\right)}\right)=(-1)^{d+j} d ! \frac{k_{j}}{\left(\begin{array}{c}
d \\
j
\end{array}\right)} .
$$

Example 5.2. Let $H(5,3)$ be a Hamming scheme over $\mathbb{F}_{3}^{5}$. Then, the first eigenmatrix $\mathbf{P}=\left(p_{j}(i)\right)$ is as follows:

$$
\left(\begin{array}{cccccc}
1 & 10 & 40 & 80 & 80 & 32 \\
1 & 7 & 16 & 8 & -16 & -16 \\
1 & 4 & 1 & -10 & -4 & 8 \\
1 & 1 & -5 & -1 & 8 & -4 \\
1 & -2 & -2 & 8 & -7 & 2 \\
1 & -5 & 10 & -10 & 5 & -1
\end{array}\right)
$$

Let $j=1$. Then we obtain the $p$-numbers $p_{1}(i)$ as the entries of the second column of the first eigenmatrix $\mathbf{P}$.

Let $L_{\text {sub }}^{\left(\beta_{1}\right)}$ be a $5 \times 6$ matrix over $\mathbb{F}_{3}^{5}$ for $\beta_{1}=\frac{(3)(1)}{5(3-1)}=-\frac{3}{10}$ as follows:

$$
\left(\begin{array}{cccccc}
1 & -6 & 8 & 0 & 0 & 0 \\
0 & 2 & -5 & 6 & 0 & 0 \\
0 & 0 & 3 & -4 & 4 & 0 \\
0 & 0 & 0 & 4 & -3 & 2 \\
0 & 0 & 0 & 0 & 5 & -2
\end{array}\right)
$$

Then, the matrices $L_{0}^{\left(\beta_{1}\right)}, L_{1}^{\left(\beta_{1}\right)}, L_{2}^{\left(\beta_{1}\right)}, L_{3}^{\left(\beta_{1}\right)}$, and $L_{4}^{\left(\beta_{1}\right)}$ are

$$
L_{0}^{\left(\beta_{1}\right)}=\left(\begin{array}{ccccc}
-6 & 8 & 0 & 0 & 0 \\
2 & -5 & 6 & 0 & 0 \\
0 & 3 & -4 & 4 & 0 \\
0 & 0 & 4 & -3 & 2 \\
0 & 0 & 0 & 5 & -2
\end{array}\right), L_{1}^{\left(\beta_{1}\right)}=\left(\begin{array}{cccc}
-5 & 6 & 0 & 0 \\
3 & -4 & 4 & 0 \\
0 & 4 & -3 & 2 \\
0 & 0 & 5 & -2
\end{array}\right)
$$




$$
L_{2}^{\left(\beta_{1}\right)}=\left(\begin{array}{ccc}
-4 & 4 & 0 \\
4 & -3 & 2 \\
0 & 5 & -2
\end{array}\right), L_{3}^{\left(\beta_{1}\right)}=\left(\begin{array}{cc}
-3 & 2 \\
5 & -2
\end{array}\right), L_{4}^{\left(\beta_{1}\right)}=\left(\begin{array}{l}
-2 \\
\end{array}\right) .
$$

Also, $\operatorname{det}\left(L_{0}^{\left(\beta_{1}\right)}\right)=240, \operatorname{det}\left(L_{1}^{\left(\beta_{1}\right)}\right)=-168, \operatorname{det}\left(L_{2}^{\left(\beta_{1}\right)}\right)=48, \operatorname{det}\left(L_{3}^{\left(\beta_{1}\right)}\right)=$ $-4, \operatorname{det}\left(L_{4}^{\left(\beta_{1}\right)}\right)=-2$. Therefore, $p_{1}(i)(i=0,1,2,3,4,5)$ are

$$
\begin{aligned}
& p_{1}(0)=(-1)^{5+1-0}\left(\begin{array}{l}
5 \\
1
\end{array}\right) \frac{0 ! \operatorname{det}\left(L_{0}^{\left(\beta_{1}\right)}\right)}{5 !}=\frac{\operatorname{det}\left(L_{0}^{\left(\beta_{1}\right)}\right)}{24}=\frac{240}{24}=10, \\
& p_{1}(1)=(-1)^{5+1-1}\left(\begin{array}{l}
5 \\
1
\end{array}\right) \frac{1 ! \operatorname{det}\left(L_{1}^{\left(\beta_{1}\right)}\right)}{5 !}=-\frac{\operatorname{det}\left(L_{1}^{\left(\beta_{1}\right)}\right)}{24}=-\frac{-168}{24}=7, \\
& p_{1}(2)=(-1)^{5+1-2}\left(\begin{array}{l}
5 \\
1
\end{array}\right) \frac{2 ! \operatorname{det}\left(L_{2}^{\left(\beta_{1}\right)}\right)}{5 !}=\frac{\operatorname{det}\left(L_{2}^{\left(\beta_{1}\right)}\right)}{12}=\frac{48}{12}=4, \\
& p_{1}(3)=(-1)^{5+1-3}\left(\begin{array}{l}
5 \\
1
\end{array}\right) \frac{3 ! \operatorname{det}\left(L_{3}^{\left(\beta_{1}\right)}\right)}{5 !}=-\frac{\operatorname{det}\left(L_{3}^{\left(\beta_{1}\right)}\right)}{4}=-\frac{-4}{4}=1, \\
& p_{1}(4)=(-1)^{5+1-4}\left(\begin{array}{l}
5 \\
1
\end{array}\right) \frac{4 ! \operatorname{det}\left(L_{4}^{\left(\beta_{1}\right)}\right)}{5 !}=\frac{\operatorname{det}\left(L_{4}^{\left(\beta_{1}\right)}\right)}{1}=\frac{-2}{1}=-2, \\
& p_{1}(5)=(-1)^{5+1-5}\left(\begin{array}{l}
5 \\
1
\end{array}\right) \frac{5 ! \operatorname{det}\left(L_{5}^{\left(\beta_{1}\right)}\right)}{5 !}=-5,
\end{aligned}
$$

respectively. Thus, $(10,7,4,1,-2,-5)^{T}$ is the first column vector of the first eigenmatrix $\mathbf{P}$.

In the following corollary, we explain a result over the Hamming scheme $H(d, q)$ as Proposition 4.3.

Corollary 5.3. For $\beta_{j}=-\frac{q j}{d(q-1)}(j=0,1, \ldots, d)$, let $\mathcal{G}_{\beta_{j}, \mathcal{N}}=u_{0}^{(j)} A_{0}+$ $u_{1}^{(j)} A_{1}+\cdots+u_{d}^{(j)} A_{d}$ be a normalized Green's function with $u_{d}^{(j)}=1$. Let $\mathbf{N}$ be a matrix with the $j$-th column vector as $\left(u_{0}^{(j)}, u_{1}^{(j)}, \ldots, u_{d}^{(j)}\right)^{T}$ for any $j=0,1, \ldots, d$ and $\beta_{j}=-\frac{q j}{d(q-1)}$. Then $u_{i}^{(j)}$ is a $(i, j)$-component of $\mathbf{N}$ and $\mathbf{N}$ is equal to $\mathbf{P D}$, where $\mathbf{D}$ is a diagonal matrix with the $(j, j)$-diagonal entries $\frac{(-1)^{j}}{\left(\begin{array}{c}d \\ j\end{array}\right)}$.

Proof. Since by Proposition 4.3, for $\beta_{j}=-\frac{q j}{d(q-1)}(j=0,1, \ldots, d)$, the $j$-th column vector of the first eigenmatrix $\mathbf{P}$ is equal to $p_{j}(d)\left(u_{0}^{(j)}, u_{1}^{(j)}, \ldots, u_{d}^{(j)}\right)^{T}$ $\left(u_{d}^{(j)}=1\right)$. Therefore,

$$
\mathbf{N}\left(\begin{array}{ccccc}
p_{0}(d) & 0 & 0 & \cdots & 0 \\
0 & p_{1}(d) & 0 & \cdots & 0 \\
0 & 0 & p_{2}(d) & \cdots & 0 \\
\vdots & \vdots & \vdots & \ddots & \vdots \\
0 & 0 & 0 & \cdots & p_{d}(d)
\end{array}\right)=\mathbf{P} .
$$


Since $p_{j}(d)=\sum_{l}(-1)^{l}(q-1)^{j-l}\left(\begin{array}{c}d \\ l\end{array}\right)\left(\begin{array}{c}0 \\ j-l\end{array}\right)=(-1)^{j}\left(\begin{array}{c}d \\ j\end{array}\right)$, we have $\mathbf{N}=\mathbf{P D}$, where $\mathbf{D}$ is a diagonal matrix with the $(j, j)$-diagonal entries $\frac{(-1)^{j}}{\left(\begin{array}{c}d \\ j\end{array}\right)}(j=0,1, \ldots, d)$.

The following theorem is a matrix version of MacWilliams identity over $H(n, q)$.

Theorem 5.4. Let $\mathcal{C}$ (respectively, $\mathcal{C}^{\perp}$ ) be a linear code (respectively, a dual code of a linear code $\mathcal{C})$ over $\mathbb{F}_{q}$ of length $d$, and let $\mathbf{a}=\left(a_{0}, a_{1}, \ldots, a_{d}\right)$ (respectively, $\left.\mathbf{b}=\left(b_{0}, b_{1}, \ldots, b_{d}\right)\right)$ be a weight distribution of $\mathcal{C}$ (respectively, $\left.\mathcal{C}^{\perp}\right)$. Then, $\mathbf{a}=\frac{1}{\left|\mathcal{C}^{\perp}\right|} \mathbf{b}\left(p_{j}(i)\right)$ is expressed by

$$
\mathrm{aD}=\frac{1}{\left|\mathcal{C}^{\perp}\right|} \mathrm{bN}
$$

where $\mathbf{N}$ is a $(d+1) \times(d+1)$ matrix with the $j$-th column vectors as $\left(u_{0}^{(j)}, \ldots\right.$, $\left.u_{d}^{(j)}\right)^{T}$ for $\beta_{j}=-\frac{q j}{d(q-1)}$, and $\mathbf{D}$ is a $(d+1) \times(d+1)$ diagonal matrix with the $(j, j)$-diagonal entries $\frac{(-1)^{j}}{\left(\begin{array}{c}d \\ j\end{array}\right)}$ for $j=0,1, \ldots, d$.

Proof. Since $\mathbf{a}=\frac{1}{\left|\mathcal{C}^{\perp}\right|} \mathbf{b P}$ and by Corollary 5.3, $\mathbf{N}=\mathbf{P D}$, so the result follows.

Example 5.5. Let $\mathbf{P}=\left(p_{j}(i)\right)$ be a first eigenmatrix of the Hamming scheme $H(5,3)$. Choosing $\beta=-\frac{3 j}{10}(j=0,1, \ldots, 5)$, we have

\begin{tabular}{c|c|c}
$j$ & a basis of $\mathcal{N}\left(L_{\text {sub }}^{\left(\beta_{j}\right)}\right)$ & $p_{j}(5)$ \\
\hline 0 & $(1,1,1,1,1,1)$ & 1 \\
1 & $\left(-2,-\frac{7}{5},-\frac{4}{5},-\frac{1}{5}, \frac{2}{5} 1\right)$ & -5 \\
2 & $\left(4, \frac{8}{5}, \frac{1}{10},-\frac{1}{2},-\frac{1}{5} 1\right)$ & 10 \\
3 & $\left(-8,-\frac{4}{5}, 1, \frac{1}{10},-\frac{4}{5}, 1\right)$ & -10 \\
4 & $\left(16,-\frac{16}{5},-\frac{4}{5}, \frac{8}{5},-\frac{7}{5}, 1\right)$ & 5 \\
5 & $(-32,16,-8,4,-2,1)$ & -1
\end{tabular}

Thus, the matrices $\mathbf{N}$ and $\mathbf{D}$ are as follows:

$$
\mathbf{N}=\left(\begin{array}{cccccc}
1 & -2 & 4 & -8 & 16 & -32 \\
1 & -\frac{7}{5} & \frac{8}{5} & -\frac{4}{5} & -\frac{16}{5} & 16 \\
1 & -\frac{4}{5} & \frac{1}{10} & 1 & -\frac{4}{5} & -8 \\
1 & -\frac{1}{5} & -\frac{1}{2} & \frac{1}{10} & \frac{8}{5} & 4 \\
1 & \frac{2}{5} & -\frac{1}{5} & -\frac{4}{5} & -\frac{7}{5} & -2 \\
1 & 1 & 1 & 1 & 1 & 1
\end{array}\right), \mathbf{D}=\left(\begin{array}{cccccc}
1 & 0 & 0 & 0 & 0 & 0 \\
0 & \frac{1}{-5} & 0 & 0 & 0 & 0 \\
0 & 0 & \frac{1}{10} & 0 & 0 & 0 \\
0 & 0 & 0 & \frac{1}{-10} & 0 & 0 \\
0 & 0 & 0 & 0 & \frac{1}{5} & 0 \\
0 & 0 & 0 & 0 & 0 & -1
\end{array}\right)
$$


Since $\mathbf{N}=\mathbf{P D}$, we have $\mathbf{N D}^{-1}=\mathbf{P}=\left(p_{j}(i)\right)$ as follows:

$$
\left(\begin{array}{cccccc}
1 & 10 & 40 & 80 & 80 & 32 \\
1 & 7 & 16 & 8 & -16 & -16 \\
1 & 4 & 1 & -10 & -4 & 8 \\
1 & 1 & -5 & -1 & 8 & -4 \\
1 & -2 & -2 & 8 & -7 & 2 \\
1 & -5 & 10 & -10 & 5 & -1
\end{array}\right)
$$

Corollary 5.6. Let $\mathcal{C}$ (respectively, $\mathcal{C}^{\perp}$ ) be a linear code (respectively, a dual code of a linear code $\mathcal{C})$ over $\mathbb{F}_{q}$ of length $d$, and let $\mathbf{a}=\left(a_{0}, a_{1}, \ldots, a_{d}\right)$ (respectively, $\left.\mathbf{b}=\left(b_{0}, b_{1}, \ldots, b_{d}\right)\right)$ be a weight distribution of $\mathcal{C}$ (respectively, $\left.\mathcal{C}^{\perp}\right)$. Then we have

$$
\sum_{i=0}^{d}\left((-1)^{d-i} \frac{i ! \operatorname{det}\left(L_{i}^{\left(\beta_{j}\right)}\right)}{d !}\right) b_{i}=(-1)^{j} \frac{\left|\mathcal{C}^{\perp}\right|}{\left(\begin{array}{c}
d \\
j
\end{array}\right)} a_{j}
$$

for $\beta_{j}=-\frac{q j}{d(q-1)},(i, j=0,1, \ldots, d)$, where $\operatorname{det}\left(L_{i}^{\left(\beta_{j}\right)}\right)$ is given as in Corollary 5.1 .

Proof. Since $\mathbf{a}=\frac{1}{\left|\mathcal{C}^{\perp}\right|} \mathbf{b}\left(p_{j}(i)\right)$, the result follows immediately from Corollary 5.1 .

\section{The Eberlein polynomial of the Johnson scheme on the normalized Green's function}

In fact, the Eberlein polynomial $E_{i}(j)$ is the $p$-number of the Johnson scheme $J(v, d)$. In this section we show that the Eberlein polynomial $E_{i}(j)$ can be explicitly computed by determinants of submatrices of $L_{s u b}^{\left(\beta_{j}\right)}$ associated with a normalized Green's function $\mathcal{G}_{\beta_{j}, \mathcal{N}}$.

Now, we introduce the Johnson scheme $J(v, d)$ as a $P$-polynomial scheme.

Johnson scheme. Let $S$ be a set of cardinality $v$ and $X=\{T \subset S:|T|=$ $d\}(d \leq v / 2)$. Define the distance of $T_{1}, T_{2} \in X$ as $d-\left|T_{1} \cap T_{2}\right|$ and let $R_{i}$ be the $i$-th distance relation on $X$, that is,

$$
R_{i}=\left\{\left(T_{1}, T_{2}\right):\left|T_{1} \cap T_{2}\right|=d-i\right\}
$$

Then $\mathfrak{X}=\left(X,\left\{R_{i}\right\}\right)(i=0,1, \ldots, d)$ is a symmetric association scheme and is called the Johnson scheme $J(v, d)$.

The Johnson scheme $J(v, d)$ is a $P$-polynomial scheme. Thus, the intersection matrix $B_{1}$ of $J(v, d)$ is a tridiagonal matrix with non-zero off-diagonal 
entries as follows:

$$
B_{1}=\left(\begin{array}{cccccc}
0 & k_{1} & & & & \\
c_{1} & a_{1} & b_{1} & & & \\
& c_{2} & a_{2} & b_{2} & & \\
& & c_{3} & a_{3} & \ddots & \\
& & & \ddots & \ddots & b_{d-1} \\
& & & & c_{d} & a_{d}
\end{array}\right),
$$

where, $a_{i}=i(v-2 i), b_{i}=(d-i)(v-d-i)$ and $c_{i}=i^{2}(i=1,2, \ldots, d)$. Thus, for $\beta_{j}=\frac{p_{1}(j)}{k_{1}}-1$, we obtain $L_{\text {sub }}^{\left(\beta_{j}\right)}$ as follows:

$$
\left(\begin{array}{cccccc}
c_{1} & a_{1}-k_{1}\left(\beta_{j}+1\right) & b_{1} & & & \\
& c_{2} & a_{2}-k_{1}\left(\beta_{j}+1\right) & b_{2} & & \\
& & c_{3} & a_{3}-k_{1}\left(\beta_{j}+1\right) & \ddots & \\
& & & \ddots & \ddots & b_{d-1} \\
& & & & c_{d} & a_{d}-k_{1}\left(\beta_{j}+1\right)
\end{array}\right) .
$$

Remark 6.1. Let $J(v, d)$ be a Johnson scheme and let $k_{i}$ and $m_{j}$ be the valencies and multiplicities of $J(v, d)$. Then

$$
k_{i}=\left(\begin{array}{l}
d \\
i
\end{array}\right)\left(\begin{array}{c}
v-d \\
i
\end{array}\right), m_{j}=\frac{v-2 j+1}{v-j+1}\left(\begin{array}{l}
v \\
j
\end{array}\right) .
$$

The following is a corollary to Theorem 4.1, and this shows that the Eberlein polynomial $E_{i}(j)$ (that is, the $p$-number over the Johnson scheme) can be explicitly computed by determinants of submatrices of $L_{s u b}^{\left(\beta_{j}\right)}$ associated with a Green's function.

Corollary 6.2. Let $J(v, d)$ be a Johnson scheme. For $\beta_{j}=\frac{j(j-v-1)}{d(v-d)}(j=$ $0,1, \ldots, d)$, let $\mathcal{G}_{\beta_{j}, \mathcal{N}}=u_{0}^{(j)} A_{0}+u_{1}^{(j)} A_{1}+\cdots+u_{d}^{(j)} A_{d}$ be a normalized Green's function of $J(v, d)$. Then, the Eberlein polynomial $E_{i}(j)(i=0,1, \ldots, d)$ is

$$
E_{i}(j)=p_{i}(j)=k_{i} \frac{u_{i}^{(j)}}{u_{0}^{(j)}}=(-1)^{i}\left(\begin{array}{c}
d \\
i
\end{array}\right)\left(\begin{array}{c}
v-d \\
i
\end{array}\right)(i !)^{2} \frac{\operatorname{det}\left(L_{i}^{\left(\beta_{j}\right)}\right)}{\operatorname{det}\left(L_{0}^{\left(\beta_{j}\right)}\right)},
$$

where $L_{i}^{\left(\beta_{j}\right)}$ is defined as $L_{i}$ in Lemma 4.5 .

Proof. We know that the Eberlein polynomial $E_{i}(j)$ is

$$
E_{i}(j)=\sum_{t=0}^{i}(-1)^{i-t}\left(\begin{array}{c}
d-t \\
i-t
\end{array}\right)\left(\begin{array}{c}
d-j \\
t
\end{array}\right)\left(\begin{array}{c}
v-d+t-j \\
t
\end{array}\right) .
$$

Thus, we have

$$
E_{1}(j)=-d+(d-j)(v-d-j+1)
$$


Since $k_{1}=d(v-d)$, we have

$$
\beta_{j}=\frac{p_{1}(j)}{k_{1}}-1=\frac{E_{1}(j)-k_{1}}{k_{1}}=\frac{j(j-v-1)}{d(v-d)} .
$$

Since $c_{i}=i^{2}$, by Remark 6.1 , the $i$-th valency $k_{i}$ of $R_{i}(i=0,1, \ldots, d)$ is

$$
k_{i}=\left(\begin{array}{l}
d \\
i
\end{array}\right)\left(\begin{array}{c}
v-d \\
i
\end{array}\right)
$$

By Theorem 4.1, the Eberlein polynomial $E_{i}(j)$ is as follows:

$$
E_{i}(j)=p_{i}(j)=(-1)^{i}\left(\begin{array}{c}
d \\
i
\end{array}\right)\left(\begin{array}{c}
v-d \\
i
\end{array}\right)(i !)^{2} \frac{\operatorname{det}\left(L_{i}^{\left(\beta_{j}\right)}\right)}{\operatorname{det}\left(L_{0}^{\left(\beta_{j}\right)}\right)}(i=0,1, \ldots, d) .
$$

Example 6.3. Let $J(8,4)$ be a Johnson scheme over $\mathbb{F}_{2}^{8}$. Then, the order of the set $X$ is 70 . Let $L_{\text {sub }}^{\left(\beta_{2}\right)}$ be a $4 \times 5$ matrix over $\mathbb{F}_{2}^{8}$ for $\beta_{2}=-\frac{14}{16}$ as follows:

$$
\left(\begin{array}{ccccc}
1 & 4 & 9 & 0 & 0 \\
0 & 4 & 6 & 4 & 0 \\
0 & 0 & 9 & 4 & 1 \\
0 & 0 & 0 & 16 & -2
\end{array}\right) .
$$

Then, the matrices $L_{0}^{\left(\beta_{2}\right)}, L_{1}^{\left(\beta_{2}\right)}, L_{2}^{\left(\beta_{3}\right)}$ and $L_{3}^{\left(\beta_{2}\right)}$ are

$$
\begin{aligned}
& L_{0}^{\left(\beta_{2}\right)}=\left(\begin{array}{cccc}
4 & 9 & 0 & 0 \\
4 & 6 & 4 & 0 \\
0 & 9 & 4 & 1 \\
0 & 0 & 16 & -2
\end{array}\right), L_{1}^{\left(\beta_{2}\right)}=\left(\begin{array}{ccc}
6 & 4 & 0 \\
9 & 4 & 1 \\
0 & 16 & -2
\end{array}\right), \\
& L_{2}^{\left(\beta_{3}\right)}=\left(\begin{array}{cc}
4 & 1 \\
16 & -2
\end{array}\right), L_{3}^{\left(\beta_{2}\right)}=(-2) .
\end{aligned}
$$

Also, $\operatorname{det}\left(L_{0}^{\left(\beta_{2}\right)}\right)=576, \operatorname{det}\left(L_{1}^{\left(\beta_{2}\right)}\right)=-72, \operatorname{det}\left(L_{2}^{\left(\beta_{2}\right)}\right)=-24, \operatorname{det}\left(L_{3}^{\left(\beta_{2}\right)}\right)=$ $-2, \operatorname{det}\left(L_{4}^{\left(\beta_{2}\right)}\right)=1$. Therefore, $E_{i}(2)(i=0,1,2,3,4)$ are as follows:

$$
\begin{aligned}
& E_{0}(2)=(-1)^{0}\left(\begin{array}{l}
4 \\
0
\end{array}\right)\left(\begin{array}{c}
8-4 \\
0
\end{array}\right) \frac{(0 !)^{2}(576)}{576}=1, \\
& E_{1}(2)=(-1)^{1}\left(\begin{array}{l}
4 \\
1
\end{array}\right)\left(\begin{array}{c}
8-4 \\
1
\end{array}\right) \frac{(1 !)^{2}(-72)}{576}=2, \\
& E_{2}(2)=(-1)^{2}\left(\begin{array}{l}
4 \\
2
\end{array}\right)\left(\begin{array}{c}
8-4 \\
2
\end{array}\right) \frac{(2 !)^{2}(-24)}{576}=-6, \\
& E_{3}(2)=(-1)^{3}\left(\begin{array}{l}
4 \\
3
\end{array}\right)\left(\begin{array}{c}
8-4 \\
3
\end{array}\right) \frac{(3 !)^{2}(-2)}{576}=2, \\
& E_{4}(2)=(-1)^{4}\left(\begin{array}{l}
4 \\
4
\end{array}\right)\left(\begin{array}{c}
8-4 \\
4
\end{array}\right) \frac{(4 !)^{2}(1)}{576}=1 .
\end{aligned}
$$


Thus, a vector $(1,2,-6,2,1)$ is the second row vector of the first eigenmatrix $\mathbf{P}$ of $J(8,4)$. In fact, the first eigenmatrix $\mathbf{P}$ of $J(8,4)$ is as follows:

$$
\left(\begin{array}{ccccc}
1 & 16 & 36 & 16 & 1 \\
1 & 8 & 0 & -8 & -1 \\
1 & 2 & -6 & 2 & 1 \\
1 & -2 & 0 & 2 & -1 \\
1 & -4 & 6 & -4 & 1
\end{array}\right)
$$

\section{References}

[1] E. Bannai and T. Ito, Algebraic Combinatorics. I, Association Schemes, Benjamin/ Cummings, Menlo Park, 1984

[2] F. Chung, PageRank as a discrete Green's function, Geometry and Analysis, I, ALM 17 (2010), 285-302.

[3] - PageRank and random walks on graphs, Fete of combinatorics and computer science, 43-62, Bolyai Soc. Math. Stud., 20, János Bolyai Math. Soc., Budapest, 2010.

[4] F. Chung and S.-T. Yau, Covering, heat kernels and spanning tree, Electron. J. Combin. 6 (1999), Research Paper 12, 21 pp.

[5] P. Delsarte, An algebraic approach to the association schemes of coding theory, Philips Res. Rep. Suppl. No. 10 (1973), vi+97 pp.

[6] C. D. Godsil, Association schemes, tech. rep., University of Waterloo, 2001.

[7] L. G. Molinari, Determinants of block tridiagonal matrices, Linear Algebra Appl. 429 (2008), no. 8-9, 2221-2226.

[8] V. S. Pless and W. C. Huffman, Fundamentals of Error-Correcting Codes, Cambridge, 2003.

[9] _ Handbook of Coding Theory Volume II, North/Holland, Elsevier, 1998.

GiL Chun Kim

Department of Mathematics

EWHA WOMANS UNIVERSITY

SEOUl 120-750, KoreA

E-mail address: higen@ewha.ac.kr

YOONJIN LEE

Department of Mathematics

EWHA Womans UNIVERSITY

SeOul 120-750, Korea

E-mail address: yoonjinl@ewha.ac.kr 